\title{
Trends in 5-, 10-, 20-, and 30-year survival rates of beta-thalassemia patients in Southern Iran, 1995-2016: A retrospective cohort study
}

\author{
Jafar Hassanzadeh, ${ }^{1}$ Alireza Mirahmadizadeh, ${ }^{2}$ Mehran Karimi, ${ }^{3}$ Yousef Veisani, ${ }^{4}$ \\ Shahab Rezaeian ${ }^{5,6}$
}

${ }^{1}$ Research Centre for Health Sciences, Department of Epidemiology, School of Health, Shiraz

University of Medical Sciences; ' 2 Department of Epidemiology, School of Health, NonCommunicable Diseases Research Center, Shiraz University of Medical Sciences; ${ }^{3}$ Hematology Research Center, Shiraz University of Medical Sciences; ${ }^{4}$ Psychosocial Injuries Research Center, Ilam University of Medical Sciences; ${ }^{5}$ Research Center for Environmental Determinants of Health, Kermanshah University of Medical Sciences, Kermanshah; ${ }^{6}$ Department of Epidemiology, School of Health, Shiraz University of Medical Sciences, Iran

\begin{abstract}
Significance for public health
A downward trend in the survival of thalassemia patients observed in this study suggest a potential supports of the community which is related with the quality of life and then improving the survival in the thalassemia patients. Moreover, some different social and individual factors might influence the survival which it is suggested further research to investigate these factors on the survival.
\end{abstract}

\begin{abstract}
Background: There is currently lack of knowledge about survival trend analysis of thalassemia patients. Therefore, the aim of the present study was to assess 5-, 10-, 20-, and 30-year survival of thalassemia patients over a 20 -year time period.

Methods: In this retrospective cohort study, we analysed the data of 982 beta-thalassemia patients in Iran. Birth cohort and traditional cohort analyses were used to obtain the 5-, 10-, 20-, and 30-year survival rates in various time intervals between 1995 and 2016.

Results: Five and 10-year survival rates remained unchanged since 1995-2016. Overall, 20- and 30-year survival rates were lower in younger birth cohorts than older ones. A declining trend was found in 20-year survival rate from 1995 to 2000 for all and also for thalassemia major patients, but was stable from 2001 to 2016. In addition, there was a declining trend in 30-year survival rate from 1995 to 2008 for all and also for thalassemia major patients, but was an increased trend from 2009 to 2016.

Conclusion: Over the past two decades and in recent birth cohorts, the 20- and 30-year survival rates has declined. In other words, declining survival trends in the birth cohorts may be associated with some different causes of mortality such as exposure to the toxic effects of iron over time and the occurrence of diseaserelated mortality.
\end{abstract}

\section{Introduction}

Thalassemia is considered as a public health challenge with an annual number of 60,000 to 70,000 new cases and 18,000 deaths worldwide. ${ }^{1}$ Thalassemia is also one of the common hemoglobinopathies disorders occurring in Iran. Despite a significant improvement in the thalassemia prevention program (TPP) ${ }^{2}$ and treatment, ${ }^{3}$ it is estimated that over 300 new cases are annually occurred in Iran. ${ }^{4}$

Conducting an obvious knowledge about epidemiological status of the disease such as incidence and survival trends can help policymaker in different aspects. This information is essential for the planning of control and prevention strategies, evaluating the effectiveness of interventions, and services delivery systems.

Previous epidemiological studies conducted on survival analysis for thalassemia disease have reported a significant improvement in the survival rate based on the comparison with other similar studies. ${ }^{5,6}$ Although, a few studies have reported a significant effect of birth cohort on the survival rate. ${ }^{7,8}$ To date, there is no evaluation of the survival trend in thalassemia patients during the past two decades, and it is unknown whether this survival trend is increasing over time or not. Therefore, the aim of the present study, which is the first in its kind and also is in line with the purposes of Iran's National Institute of Health Research, ${ }^{9}$ was to assess whether overall and 5-, 10-, 20-, and 30-year survival rates of thalassemia patients have improved over a 20 -year time period.

\section{Materials and Methods}

A retrospective cohort study was conducted on enrolling 1003 beta-thalassemia (major, intermedia, and sickle cell) patients in 2016 in Shiraz, the southern Iran. The inclusion criteria were having a definitive diagnosis of beta-thalassemia major. We have excluded the sickle cell thalassemia (16 patients) and those with missing data (5 patients) from the analysis (Figure 1). This study was performed following approval by the Ethics Review Committee of Shiraz University of Medical Sciences.

The Iranian TPP was established in $1997 .^{10}$ There is a referral Hospital with a document-based system in each county for treatment services of the patients. Data were extracted from patients' medical records using a comprehensive predetermined checklist, ${ }^{5}$ which the information including type of thalassemia, date of birth, and date of death (if applicable) were used in this analysis.

We derived 5-, 10-, 20-, and 30-year survival rates (as the endpoints of this study) in various time intervals between 1995 and 2016. The survival time for the patients was considered as the interval between the date of birth and the date of death or May 31 in each year during 1995-2016. The Cochran-Armitage test was used for survival trend analysis. The Chi-square for trend test was used to compare in survival trends by type of thalassemia. The 
subjects were divided into four birth cohorts (1961-70, 1971-80, 1981-90, and 1991-2000) and 20-, and 30-year survival rates were calculated in various time intervals between 1995 and 2016 for each birth cohort.

Moreover, the Kaplan-Meier estimate and log-rank tests were used to describe and compare overall survival based on different birth cohorts (1970s and earlier, 1980s, 1990s, and 2000 to the present) which birth cohort 1970s was considered to be the reference group. All statistical analyses were done with 95\% confidence interval using Stata 11.2 (StataCorp. College Station, TX, USA).

\section{Results}

The data of 982 patients were identified. Of them, 704 (72\%) patients were beta thalassemia major and 227 patients died.

Table 1 shows trend of 30 -year survival rates of the patients by birth cohorts in the past two decades. Overall, 30-year survival rate is lower in younger birth cohorts than older ones in 2010 to 2016 for which the survival data for three birth cohorts are available. In other words, 30-year survival rate for birth cohort 1981-90 at year 2016 was 0.69 which for birth cohort $1961-70$ at year 2016 was 0.882 .

Trend of 20-year survival rates of the patients by birth cohorts in the past two decades is shown in Table 2 . In all investigated year (calendar year), the 20-year survival rate slowly declined with increasing birth cohort. For example, 20-yer survival rate for birth cohort 1961-70, 1971-80, 1981-90, and 1991-2000 was 100, 95.2, 84.3 , and $83.0 \%$, respectively (Figure 2 ).

Five-, 10-, 20-, and 30-year survival estimates for all thalassemia patients and by type of thalassemia disease obtained in

Table 1. Trend of 30-year survival rates of thalassemia patients by birth cohorts in the past two decades, Iran.

\begin{tabular}{lccc} 
Calendar year & \multicolumn{3}{c}{ Birth cohorts } \\
& $1961-70$ & $1971-80$ & $1981-90$ \\
1996 & 1.00 & - & - \\
1997 & 0.926 & - & - \\
\hline 1998 & 0.862 & - & - \\
1999 & 0.875 & - & - \\
2000 & 0.882 & 0.873 & - \\
2001 & 0.882 & 0.878 & - \\
\hline 2002 & 0.882 & 0.857 & - \\
2003 & 0.882 & 0.861 & - \\
2004 & 0.882 & 0.837 & - \\
2005 & 0.882 & 0.737 & - \\
\hline 2006 & 0.882 & 0.737 & - \\
2007 & 0.882 & 0.734 & - \\
2008 & 0.882 & 0.730 & - \\
2009 & 0.882 & 0.742 & - \\
\hline 2010 & 0.882 & 0.744 & 0.628 \\
2011 & 0.882 & 0.752 & 0.630 \\
\hline 2012 & 0.882 & 0.752 & 0.644 \\
2013 & 0.882 & 0.752 & 0.641 \\
\hline 2014 & 0.882 & 0.752 & 0.661 \\
2015 & 0.882 & 0.752 & 0.679 \\
\hline 2016 & 0.882 & 0.752 & 0.690 \\
\hline & & & \\
\hline & & & - \\
\hline
\end{tabular}

various time intervals are presented in Figure 3 and 4, respectively. In the Figures, the 5-, and 10-year survival rates remained
Total patients included in the cohort ( $\mathrm{n}=1003$ beta-thalassemia)

Excluded data:

Sickle cell thalassemia: $n=16$

Missing data: $\mathrm{n}=5$

Total patients included in the survival analysis $(\mathrm{n}=982)$

Patients with beta thalassemia major $=704$

Patients with beta thalassemia intermedia $=278$

Patients were died $=227$

\begin{tabular}{|l|l|l|l|}
\hline $\begin{array}{l}\text { Birth cohort } \\
1961-70 \\
n=18\end{array}$ & \begin{tabular}{|l|l} 
Birth cohort \\
$1971-80$ \\
$n=105$
\end{tabular} & $\begin{array}{l}\text { Birth cohort } \\
1981-90 \\
n=757\end{array}$ \\
\hline
\end{tabular}

Figure 1. Flowchart of this retrospective study.

Table 2. Trend of 20-year survival rates of thalassemia patients by birth cohorts in the past two decades, Iran

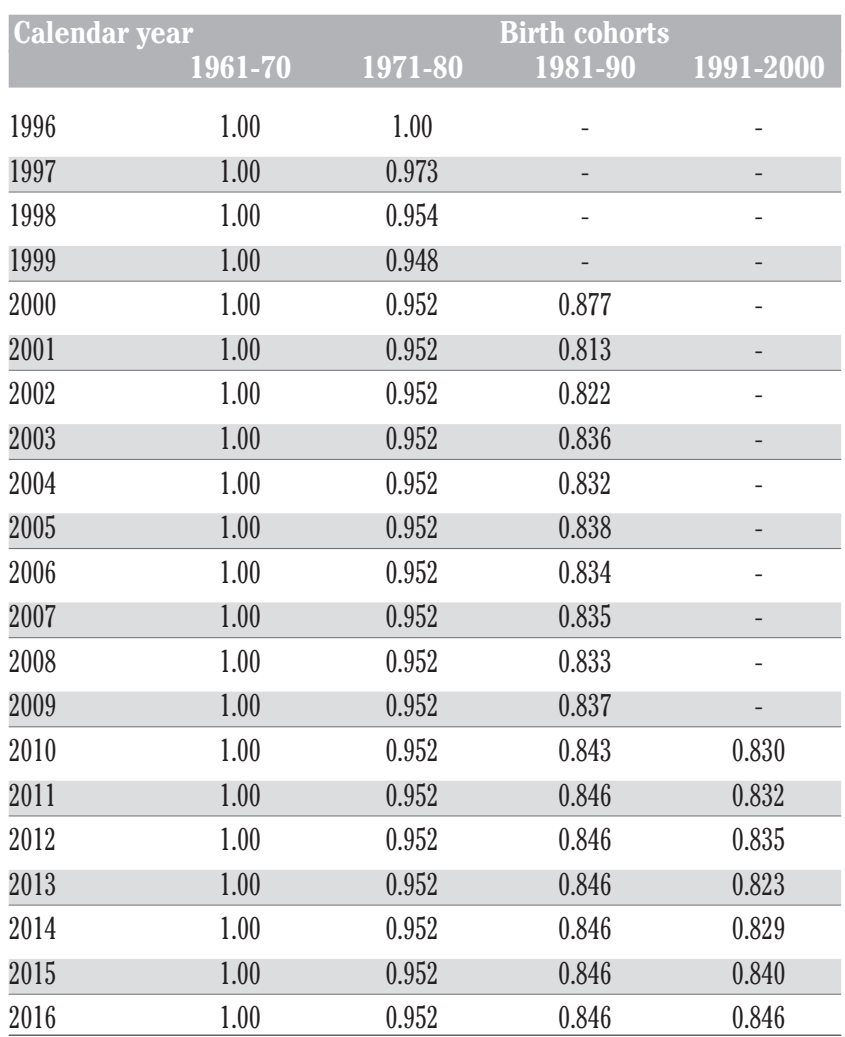


unchanged since 1995-2016. There was a declining trend in 20year survival rate from 1995 to 2000 for thalassemia major patients, but was stable from 2001 to 2016 . Also, a stable trend was observed for 20-year survival rate in thalassemia intermedia patients. In addition, there was a declining trend in 30-year survival rate from 1995 to 2008 for all and also for thalassemia major patients, but was an increased trend from 2009 to 2016 for both types of thalassemia.

\section{Discussion}

Based on data collected from a large thalassemia patients' cohort, we found a new finding which was inconsistent with finding from the previous survival studies worldwide. We found a declining trend in the 20- and 30-year survival rates from both TM and TI patients during 1995-2016. But, the 5- and 10-year survival rates were stable during two past decades. In this study, birth cohort effect was also investigated on the 20- and 30-year survival rates. Our finding showed that older birth cohorts had a higher 20and 30-year survival rates.

The 20- and 30-year survival rates have decreased during two past decades since 1995 for both TM and TI patients. The trend of both 20 - and 30-year survival rates had a sharp reduction before 2000. There are some reasons which could explain this finding.
After implementation of the thalassemia prevention program from 1997, the quality of healthcare services may be improved. On the other hand, several reforms have been implemented to Iran's health system over the last three decades. For example, the establishment

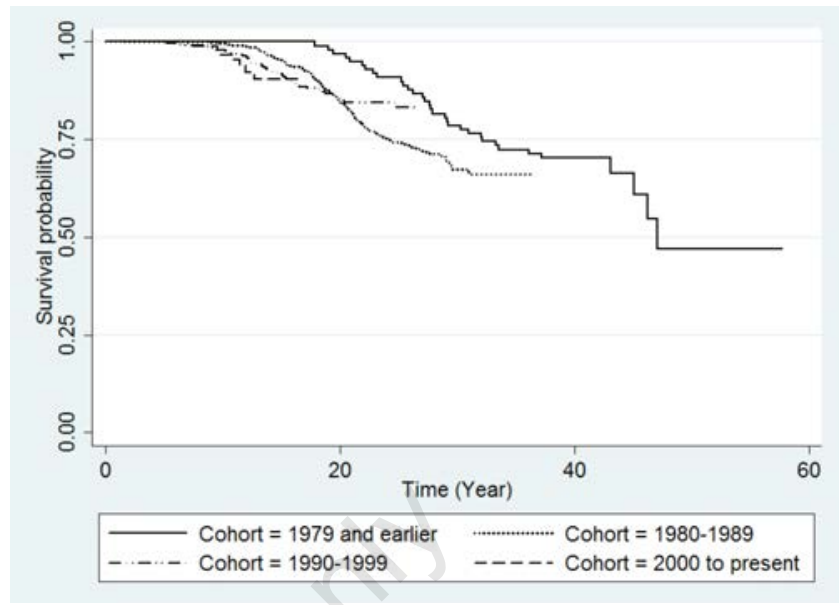

Figure 2. Kaplan-Meier survival curves for the thalassemia patients enrolled in this study stratified by birth cohorts.

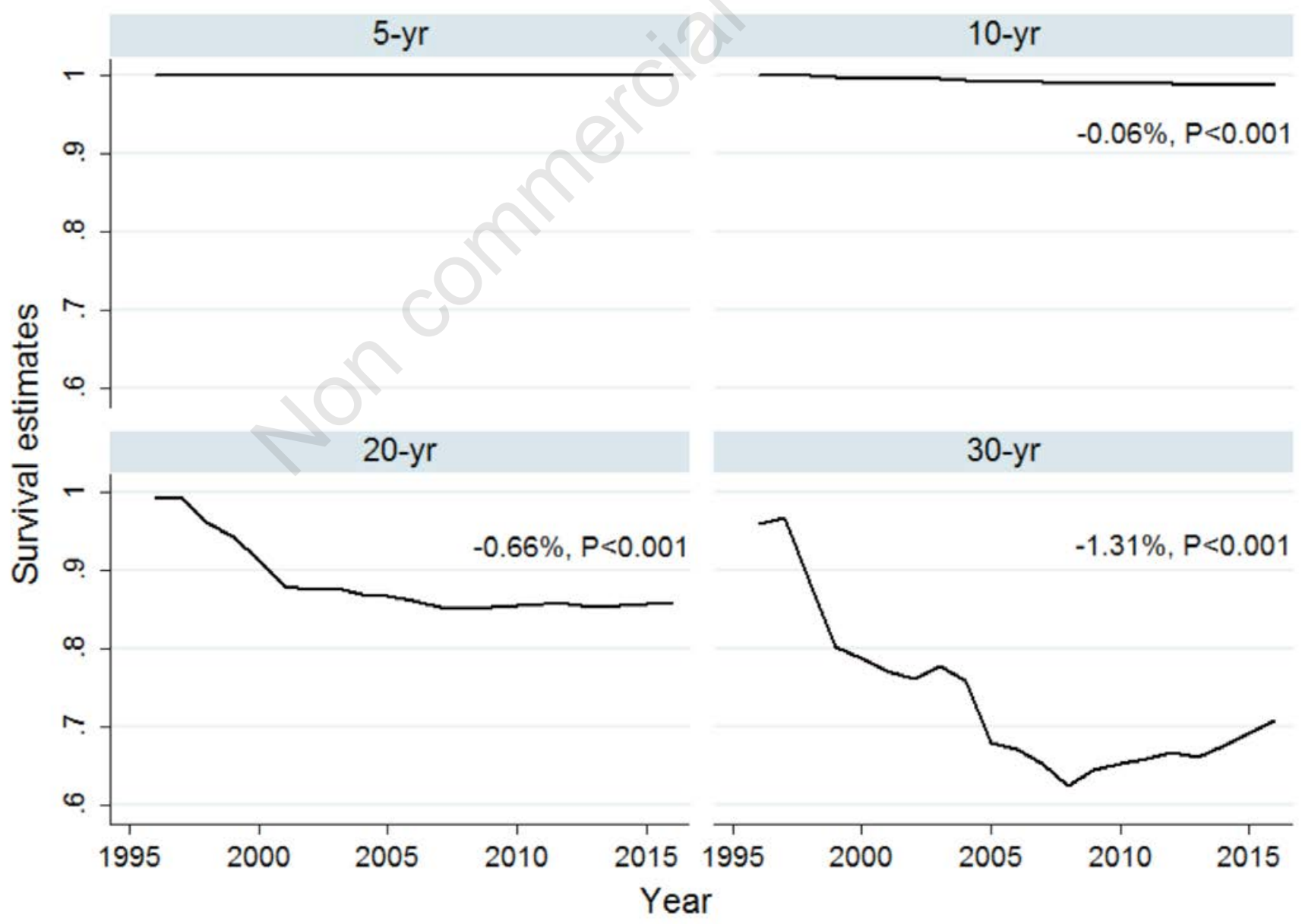

Figure 3. Five-, 10-, 20-, and 30-year survival estimates for thalassemia patients obtained in various time intervals, Iran, $1995-2016$. 
of the Family Physician Programme in $2005,{ }^{11}$ and the Health Sector Evolution Plan in $2014^{12}$ which the purposes of them were to improve the referral system and provide healthcare services in rural and urban areas. These reforms could explain the stable trend in the 20-year survival rate after 2000 and increased trend in 30year survival rate after 2008 (Figure 3).

The 20- and 30-year survival rates declined with increasing birth cohort, which the birth cohort 1961-70 had better survival rates than birth cohort 1971 to present. We found no similar study for comparison. But the probable explanation for this is that older patients that survive from bone marrow transplantation (BMT) had very good chance of long term survival as they do not require blood transfusion. Therefore, selection bias may be affected of this finding. Another explanation is that in most previous survival studies, cohort data were cross-sectionally analysed to obtain the survival rate at the end of study period. In these studies, Kaplan-Meier plots were used to compare different birth cohorts which are inconsistent with our findings based on birth cohort. Telfer et al. ${ }^{13}$ conducted a study to identify factors associated with long time survival in thalassemia patients. They reported that improved survival after year 2000 compared to 1999 and earlier was unexpected, because, patients born before 1999 have more exposed to the toxic effects of iron-chelation therapy and then have more vulnerable to the heart failure. Other studies have reported that some factors such as delay in registration of thalassemia patients in the disease registration system, ${ }^{2,4}$ and delayed diagnosis of the patients ${ }^{14}$ may affect the poor outcomes for thalassemia patients. Another study conducted by Ladis et al. ${ }^{15}$ showed a higher survival rate for patients born after 1975 compared to those born before. Wu et al. ${ }^{16}$ recently published a study to determine the survival rate and complications in TM patients and fund an improved survival rate from $89.6 \%$ of the patients born before 1995 to $94.3 \%$ of those born after 1995.

Based on the traditional Kaplan-Meier survival analysis, our findings have also shown a significant difference among birth cohorts (Figure 4). In other words, older birth cohorts had a higher survival rate. This result is inconsistent with the results from other survival studies. ${ }^{7,8,15}$ In a study with 1044 Greek thalassemia to assess the survival rate compared to the general population, authors found that birth cohort has a negative significant trend towards past decades. ${ }^{8}$

Despite the growing success rate of thalassemia prevention programs in the past two decades, ${ }^{2,17}$ questions remain as to why the survival rate of the patients has declined. In our opinion, establishment a multicentre study could help to identify the underlying causes of this finding.

The data used in our study have been extracted two times from document-based database. First time for another objective, ${ }^{18}$ which was approved by Fasa University of Medical Sciences (Grant No. 93188) and the second time for our purpose. Therefore, the quality

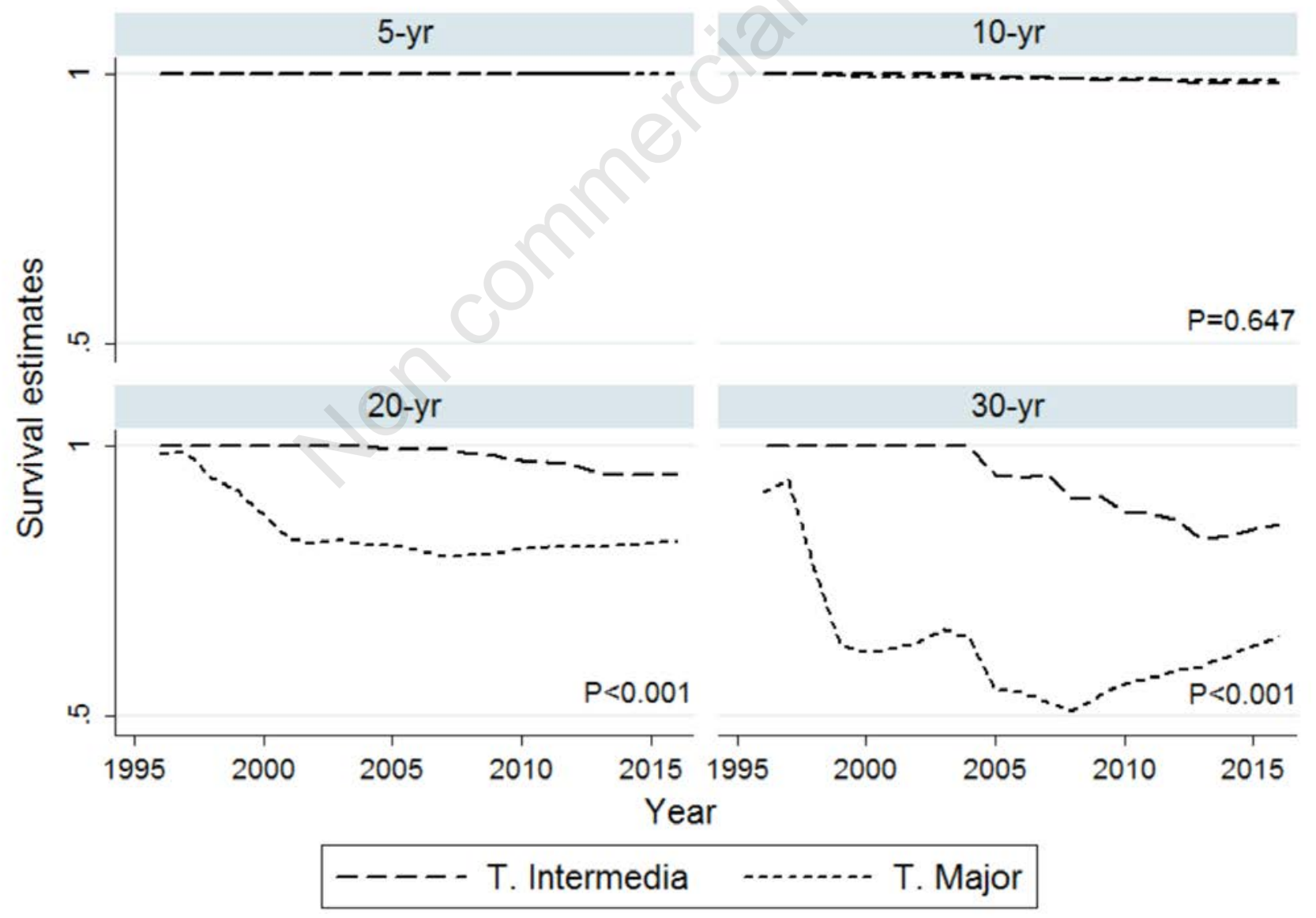

Figure 4. Five-, 10-, 20-, and 30-year survival estimates by type of thalassemia disease obtained in various time intervals, Iran, 19952016. 
and precision of the data was a significant strength of this study. An important limitation of the study was the lack of causes of death in the studied cohort. This information could have helped in the better inference of the declining trend in the 20-and 30-year survival rates.

Correspondence: Shahab Rezaeian, Research Center for Environmental Determinants of Health, Kermanshah University of Medical Sciences, P.0. Box 6719851351, Kermanshah, Iran.

Tel.: +98.9189814634 - Fax: +98.8338263048 .

E-mail: shahab.rezayan@gmail.com

Key words: Survival analysis, Beta thalassemia, Cohort Studies, Iran.

Acknowledgments: This work was extracted from the PhD thesis by Shahab Rezaeian and was financially supported by Shiraz University of Medical Sciences (Grant No. 94-7610). The authors would like to thank Mr. H. R. Tabatabaee (Scopus Author ID: 6507314325) for providing us a number of valuable comments.

Contributions: Study concept and design: JH, SR. Analysis and interpretation of data: JH, SH, AM. Drafting of the manuscript: JH, SR. Critical revision of the manuscript for important intellectual content: JH, AM, MK, YV. Statistical analysis: SR, JH. Study supervision: JH.

Conflict of interest: The authors declare no potential conflict of interest.

Received for publication:

Accepted for publication:

(C) Copyright xxx et al., 2017

Licensee PAGEPress, Italy

Journal of Public Health Research 2017;6:1001

doi:10.4081/jphr.2017.1001

This work is licensed under a Creative Commons Attribution NonCommercial 4.0 License (CC BY-NC 4.0).

\section{Conclusions}

Over the past two decades and in recent birth cohorts, the 20and 30-year survival rates has declined. In other words, declining survival trends in the birth cohorts may be associated with some different causes of mortality such as exposure to the toxic effects of iron over time and the occurrence of disease-related mortality. Moreover, some different social and individual factors might influence the declining trends which further research to investigate these factors on the survival trend is suggested.

\section{References}

1. Lozano R, Naghavi M, Foreman K, et al. Global and regional mortality from 235 causes of death for 20 age groups in 1990 and 2010: a systematic analysis for the Global Burden of
Disease Study 2010. Lancet 2012;380:2095-128.

2. Miri M, Tabrizi Namini M, Hadipour Dehshal M, et al. Thalassemia in Iran in last twenty years: the carrier rates and the births trend. Iran J Blood Cancer 2013;6:11-8.

3. Keikhaei B, Yousefi H, Bahadoram M. Clinical and haematological effects of hydroxyurea in beta-thalassemia intermedia patients. J Clin Diagn Res 2015;9:1-3.

4. Dehshal MH, Ahmadvand A, Darestani SY, et al. Secular trends in the national and provincial births of new thalassemia cases in Iran from 2001 to 2006. Hemoglobin 2013;37:124-37.

5. Rajaeefard A, Hajipour M, Tabatabaee HR, et al. Analysis of survival data in thalassemia patients in Shiraz, Iran. Epidemiol Health 2015;37:e2015031.

6. Zamani R, Khazaei S, Rezaeian S. Survival analysis and its associated factors of beta thalassemia major in Hamadan Province. Iran J Med Sci 2015;40:233-9.

7. Borgna-Pignatti C, Rugolotto S, De Stefano P, et al. Survival and complications in patients with thalassemia major treated with transfusion and deferoxamine. Haematologica 2004;89:1187-93.

8. Ladis V, Chouliaras G, Berdoukas V, et al. Survival in a large cohort of Greek patients with transfusion-dependent beta thalassaemia and mortality ratios compared to the general population. Eur J Haematol 2011;86:332-8.

9. I.R. Iran's National Institute of Health Research. 2015.

10. Samavat A, Modell B. Iranian national thalassaemia screening programme. BMJ 2004;329:1134-7.

11. Hatam N, Joulaei H, Kazemifar Y, Askarian M. Cost efficiency of the family physician plan in fars province, southern iran. Iran J Med Sci 2012;37:253-9.

12. Moradi-Lakeh M, Vossogh-Moghaddam A. Health sector evolution plan in Iran; equity and sustainability concerns. Int J Health Policy Manag 2015;4:637-40.

13. Telfer P, Coen PG, Christou S, et al. Survival of medically treated thalassemia patients in Cyprus. Trends and risk factors over the period 1980-2004. Haematologica 2006;91:1187-92.

14. Hassanzadeh J, Mirahmadizadeh A, Karimi M, Rezaeian S. Delay in diagnosis of hemoglobulinopathies (thalassemia, sickle cell anemia): a need for management of thalassemia programs. Iran J Pediatr 2016;27:e6740.

15. Ladis V, Chouliaras G, Berdousi H, et al. Longitudinal study of survival and causes of death in patients with thalassemia major in Greece. Ann N Y Acad Sci 2005; 1054:445-50.

16. Wu HP, Lin CL, Chang YC, et al. Survival and complication rates in patients with thalassemia major in Taiwan. Pediatr Blood Cancer 2017;64:135-8.

17. Hashemieh M, Timori Naghadeh H, Tabrizi Namini M, et al. The Iran thalassemia prevention program: success or failure? Iran J Ped Hematol Oncol 2015;5:161-6.

18. Hajipour M, Soltani M, Najafifar R, et al. Complications of beta-thalassemia patients and their related factors in Iran. Int J Dev Res 2015;5:5009-14 